\title{
Espacio literario relevante sobre el problema del vendedor viajero (TSP): contenido, clasificación, métodos y campos de inspiración
}

\author{
Jorge Ivan Pérez Rave ${ }^{\mathrm{a}}$, Gloria Patricia Jaramillo Álvarez ${ }^{\mathrm{b}}$ \\ a*ejipr056@udea.edu.co, Universidad de Antioquia, Colombia \\ bypjarami@unal.edu.co, Universidad Nacional de Colombia, Colombia
}

\begin{abstract}
Resumen
Se describe y se analiza un espacio literario relevante sobre el Problema del Vendedor Viajero (TSP) en términos de contenido, clases de TSP, métodos y campos de inspiración. Los datos empleados provinieron de los trabajos más citados en Scopus sobre el TSP, tanto a través de la historia como en el período 2006-2010. Se encontró que el TSP prevalece en las investigaciones, con enfoques tanto en el problema original como en sus variantes, entre las cuales se identificaron el TSP Múltiple y el TSP Probabilístico. Entre los principales campos de inspiración para resolver el TSP están la evolución biológica y su base genético-molecular, el comportamiento de hormigas reales, la termodinámica, las estrategias sistemáticas para combinar reglas de decisión y la búsqueda de vecindades. Hoy día se tiende a desarrollar métodos híbridos, especialmente integrando enfoques globales con búsquedas locales, y se identifica la necesidad de introducir nuevos campos de inspiración.
\end{abstract}

Palabras clave

Problema del vendedor viajero. Revisión sistemática. Métodos heurísticos. Optimización combinatoria.

\section{Introducción}

Este artículo tiene por objetivo describir y analizar el espacio literario relevante sobre el Problema del Vendedor Viajero (TSP, sigla en inglés para Travelling Salesman Problem), identificado en Pérez (2011) a través de una aproximación a la revisión sistemática, extrapolada desde los campos de la salud (DAVIS et al., 1995) y de experiencias recientes en ingeniería de software (STAPLES; NIAZI, 2007; KITCHENHAM et al., 2009). Pérez (2011) inició con la definición de las preguntas de investigación, asociadas con la identificación de la vigencia del tema y las fuentes literarias más citadas por la comunidad científica, a través de la historia y, específicamente, entre 2006 y 2010. Para responder a dichas preguntas, delimitó un espacio de búsqueda planeado, estructurado y basado en datos provenientes de Scopus. El foco del presente artículo es profundizar en el espacio literario anteriormente mencionado, relevante para el estudio del TSP. Ese espacio representa, bajo los criterios de búsqueda, sólo el 1,9\% del total de documentos que tratan el TSP como eje central (tema o problema de prueba principales) y reúne el $68 \%$ de las citaciones científicas (PÉREZ, 2011).

Además, haciendo énfasis en la revisión de tipo sistemática, vale notar que de los 54.124 documentos encontrados en Scopus, al 15-02-2011 (21:22hrs), bajo el término "systematic review", solo el 1,2\% pertenece a las áreas de Ingeniería, Ciencias de la Computación y Ciencias de la Decisión, de lo cual se deduce la oportunidad de hacer contribuciones en el ámbito de la investigación de operaciones.

Por lo expuesto, y continuando con las necesidades de estudio planteadas en Pérez (2011), se buscó responder a las siguientes preguntas de investigación: 1) ¿De qué tratan las diez publicaciones más citadas, a lo largo de la historia, que abordan el TSP como eje central? 2) ¿De qué tratan las diez publicaciones 
más citadas en el periodo 2006-2010, que abordan el TSP como eje central?; 3) ¿Qué clases de TSP fueron abordados en los estudios más citados sobre el tema través de la historia y entre 2006 y 2010?; 4) ¿Qué métodos de resolución fueron empleados en los estudios más citados sobre el TSP?; y 5) ¿Qué campos inspiraron los métodos de resolución empleados en los estudios más citados sobre el TSP?

\section{Materiales y métodos}

Para responder las preguntas de investigación se analizaron datos pertenecientes al espacio literario relevante sobre el TSP, identificado en Pérez (2011), quien contempló los términos: "traveling salesman problem”, "travelling salesman problem”, "traveling salesperson problem" y "travelling salesperson problem”, circunscriptos a la Ingeniería, Ciencias de la Computación y Ciencias de la Decisión. La búsqueda fue realizada por Pérez (2011) el 17/02/2011 en Scopus, una de las principales bases de datos científicos (CODINA, 2005). Algunos de los criterios para la inclusión de manuscritos en Scopus son: que las revistas de donde provengan empleen revisión por árbitros, conformidad con normas técnicas de calidad, que contengan resúmenes en inglés y que sean oportunas en la edición. El procedimiento seguido por Pérez (2011) constó de selección de términos de búsqueda, delimitación del espacio, trabajo de campo (búsqueda propiamente dicha), control de calidad de los trabajos identificados, depuración de datos y reporte de los resultados. De esta manera, recurriendo a principios de la revisión sistemática, puede verse que la identificación de los estudios no estuvo sesgada por los intereses del revisor.

Los insumos empleados en el presente artículo fueron: para responder la pregunta 1, las diez publicaciones más citadas sobre el TSP a través de la historia (Tabla 1) y, para responder a la pregunta 2, las diez publicaciones más citadas sobre el tema entre 2006 y 2010 (Tabla 2). Para responder las preguntas 3 a 5 se construyó una tabla-resumen (Tabla 3), fruto del análisis de los resultados de las preguntas 1 y 2 .

\section{Resultados}

\section{1. ¿De qué tratan las diez publicaciones más citadas a lo largo de la historia, que abordan como eje central el TSP?}

Held y Karp (1971) exponen un procedimiento tipo ramificación y acotamiento que parte del estudio de la relación entre el TSP y el problema de árbol de mínima expansión. Junto con un primer estudio en 1970, este segundo trabajo introduce la relajación

Tabla 1. Las diez publicaciones más citadas de la historia que tuvieron al TSP como eje central (tema o problema principal de prueba).

\begin{tabular}{|c|c|c|c|c|c|c|}
\hline Posición & Título & Autor(es) & Año & Revista & Tipo & Citas \\
\hline 1 & $\begin{array}{l}\text { Ant colony system: A cooperative learning } \\
\text { approach to the traveling salesman problem }\end{array}$ & $\begin{array}{l}\text { Dorigo, M.; } \\
\text { Gambardella, L. }\end{array}$ & 1997 & $\begin{array}{l}\text { IEEE Transactions } \\
\text { on Evolutionary } \\
\text { Computation }\end{array}$ & Artículo & 1,946 \\
\hline 2 & $\begin{array}{c}\text { Thermodynamical approach to the traveling } \\
\text { salesman problem: An efficient simulation } \\
\text { algorithm }\end{array}$ & Cerný, v. & 1985 & $\begin{array}{l}\text { Journal of } \\
\text { Optimization Theory } \\
\text { and Applications }\end{array}$ & Artículo & 635 \\
\hline 3 & TSPLIB. A traveling salesman problem library & Reinelt, G. & 1991 & $\begin{array}{l}\text { ORSA journal on } \\
\text { computing }\end{array}$ & Artículo & 443 \\
\hline 4 & $\begin{array}{c}\text { The traveling-salesman problem and minimum } \\
\text { spanning trees: Part } 11\end{array}$ & Held, M.; Karp, R. & 1971 & $\begin{array}{l}\text { Mathematical } \\
\text { Programming }\end{array}$ & Artículo & 161 \\
\hline 5 & $\begin{array}{c}\text { MAX-MIN Ant System and local search for the } \\
\text { traveling salesman problem }\end{array}$ & $\begin{array}{l}\text { Stuetzle, T.; } \\
\text { Hoos, H. }\end{array}$ & 1997 & $\begin{array}{l}\text { Proceedings of the } \\
\text { IEEE Conference } \\
\text { on Evolutionary } \\
\text { Computation, ICEC }\end{array}$ & $\begin{array}{l}\text { Articulo en } \\
\text { Conferencia }\end{array}$ & 154 \\
\hline 6 & $\begin{array}{l}\text { Fast algorithms for geometric traveling } \\
\text { salesman problems }\end{array}$ & Bentley, J. & 1992 & $\begin{array}{l}\text { ORSA journal on } \\
\text { computing }\end{array}$ & Artículo & 128 \\
\hline 7 & $\begin{array}{l}\text { Self-organizing feature maps and the } \\
\text { travelling salesman problem }\end{array}$ & $\begin{array}{l}\text { Angéniol, B.; De } \\
\text { La Croix V.; Le } \\
\text { Texier, J. }\end{array}$ & 1988 & Neural Networks & Artículo & 107 \\
\hline 8 & $\begin{array}{c}\text { The traveling salesman problem: An overview } \\
\text { of exact and approximate algorithms }\end{array}$ & Laporte, G. & 1992 & $\begin{array}{l}\text { European Journal of } \\
\text { Operational Research }\end{array}$ & Artículo & 98 \\
\hline 9 & $\begin{array}{l}\text { Applying evolutionary programming to } \\
\text { selected traveling salesman problems }\end{array}$ & Fogel, D. & 1993 & $\begin{array}{l}\text { Cybernetics and } \\
\text { Systems }\end{array}$ & Artículo & 97 \\
\hline 10 & $\begin{array}{c}\text { Genetic algorithms for the travelling salesman } \\
\text { problem: A review of representations and } \\
\text { operators }\end{array}$ & Larrañaga, P. et al. & 1999 & $\begin{array}{l}\text { Artificial Intelligence } \\
\text { Review }\end{array}$ & Artículo & 96 \\
\hline
\end{tabular}


Tabla 2. Las diez publicaciones más citadas entre 2006-2010 que tuvieron al TSP como eje central (tema o problema principal de prueba).

\begin{tabular}{|c|c|c|c|c|c|c|}
\hline Posición & Título & Autor(es) & Año & Revista & Tipo & Citas \\
\hline 1 & $\begin{array}{c}\text { The multiple traveling salesman } \\
\text { problem: An overview of formulations } \\
\text { and solution procedures }\end{array}$ & Bektas, T. & 2006 & Omega & Revisión & 48 \\
\hline 2 & $\begin{array}{l}\text { A random-key genetic algorithm for the } \\
\text { generalized traveling salesman problem }\end{array}$ & $\begin{array}{l}\text { Snyder, L.; } \\
\text { Daskin, M. }\end{array}$ & 2006 & $\begin{array}{l}\text { European Journal of Operational } \\
\text { Research }\end{array}$ & Artículo & 46 \\
\hline 3 & $\begin{array}{c}\text { Implementation of an effective hybrid } \\
\text { GA for large-scale traveling salesman } \\
\text { problems }\end{array}$ & $\begin{array}{l}\text { Nguyen, } \mathrm{H} \text {. } \\
\text { et al. }\end{array}$ & 2007 & $\begin{array}{l}\text { IEEE Transactions on Systems, Man, } \\
\text { and Cybernetics, Part B: Cybernetics }\end{array}$ & Artículo & 32 \\
\hline 4 & $\begin{array}{l}\text { A hybrid scatter search for the } \\
\text { probabilistic traveling salesman problem }\end{array}$ & Liu, Y. & 2007 & Computers and Operations Research & Artículo & 23 \\
\hline 5 & $\begin{array}{c}\text { A new approach to solving the multiple } \\
\text { traveling salesperson problem using } \\
\text { genetic algorithms }\end{array}$ & $\begin{array}{l}\text { Carter, A.; } \\
\text { Ragsdale, C. }\end{array}$ & 2006 & $\begin{array}{c}\text { European Journal of Operational } \\
\text { Research }\end{array}$ & Artículo & 21 \\
\hline 6 & $\begin{array}{l}\text { Variable neighborhood search for the } \\
\text { pickup and delivery traveling salesman } \\
\text { problem with LIFO loading }\end{array}$ & $\begin{array}{l}\text { Carrabs, F.; } \\
\text { Cordeau, J.; } \\
\text { Laporte, G. }\end{array}$ & 2007 & INFORMS Journal on Computing & Artículo & 18 \\
\hline 7 & $\begin{array}{c}\text { A Compressed-Annealing Heuristic for } \\
\text { the Traveling Salesman Problem with } \\
\text { Time Windows }\end{array}$ & $\begin{array}{l}\text { Ohlmann, J.; } \\
\text { Thomas, B. }\end{array}$ & 2007 & INFORMS Journal on Computing & Artículo & 16 \\
\hline 8 & $\begin{array}{l}\text { Aggregation for the probabilistic } \\
\text { traveling salesman problem }\end{array}$ & Campbell, A. & 2006 & Computers and Operations Research & Artículo & 15 \\
\hline 9 & $\begin{array}{c}\text { Traveling salesperson problems for the } \\
\text { Dubins vehicle }\end{array}$ & $\begin{array}{l}\text { Savla, K.; } \\
\text { Frazzoli, E.; } \\
\text { Bullo, F. }\end{array}$ & 2008 & $\begin{array}{c}\text { IEEE Transactions on Automatic } \\
\text { Control }\end{array}$ & Artículo & 14 \\
\hline 10 & $\begin{array}{c}\text { Hybrid ant colony optimization using } \\
\text { memetic algorithm for traveling } \\
\text { salesman problem }\end{array}$ & $\begin{array}{l}\text { Duan, H.; } \\
\text { Yu, X. }\end{array}$ & 2007 & $\begin{array}{l}\text { Proceedings of the } 2007 \text { IEEE } \\
\text { Symposium on Approximate } \\
\text { Dynamic Programming and } \\
\text { Reinforcement Learning, ADPRL }\end{array}$ & $\begin{array}{l}\text { Artículo en } \\
\text { Conferencia }\end{array}$ & 13 \\
\hline
\end{tabular}

Fuente: Pérez (2011).

lagrangiana probada en hasta 64 ciudades. Cerný (1985) propone la resolución de problemas de optimización combinatoria a través de una analogía con el campo de la termodinámica, probada en el TSP, permutando aleatoriamente las estaciones del viajero con una probabilidad en función del tamaño de la ruta. Este trabajo, aunque no atribuyéndosele como único proponente, introduce el método de Recocido Simulado, obteniendo resultados satisfactorios en instancias de hasta 200 ciudades; en algunos casos arrojando la solución óptima y, en otros, acercándose a ella. Angéniol, De La Croix y Le Texier (1988) presentan un algoritmo para abordar el TSP basado en los mapas auto-organizados de Kohonen, validando esta red neuronal en una instancia de 1.000 ciudades.

Reinelt (1991) comparte con la comunidad la librería TSP (TSPLIB), exponiéndola como una colección de problemas del agente viajero, cuyo fin es proporcionar a los investigadores de todo el mundo una amplia gama de instancias TSP, agregando para cada problema su descripción y límites, y haciendo referencia, además, a las pruebas de cómputo realizadas para algunos de los problemas. Laporte (1992) expone una recopilación de algunos de los principales algoritmos exactos y de aproximación para el TSP.
Entre los algoritmos exactos trata aquellos basados en la formulación de programación lineal entera y en árboles generadores. Entre los algoritmos de aproximación, trata heurísticas de construcción como el Vecino más Cercano o como las inserciones (más cercana, más barata, etc.), también heurísticas de mejoramiento, como el $k$-opt, y finalmente, heurísticas combinadas.

Bentley (1992) analiza diversos métodos rápidos de resolución del TSP, entre ellos: vecino más cercano, inserción más lejana, heurísticas basadas en árboles y optimizadores locales (2-opt, 2H-opt, 3-opt); los implementa y los compara computacionalmente a nivel de eficiencia, robustez y la función que siguen. Los tres optimizadores locales fueron incorporados en los demás métodos a fin de analizar sus comportamientos. Destaca la eficiencia práctica del método del Vecino más Cercano, múltiples fragmentos, partición recursiva rápida y habilidad de incorporar 2-opt y $2 \mathrm{H}$-opt en múltiples fragmentos, probados en un millón de ciudades. Fogel (1993) destaca la inspiración en la evolución natural para el diseño de algoritmos de optimización de búsqueda estocástica, entre ellos, la programación evolutiva, probada en diversas instancias del TSP, obteniendo con 30, 50 y 75 ciudades, 
resultados iguales o mejores que los reportados hasta ese entonces; con 1.000 ciudades, la solución excedió en un 5\% al óptimo esperado.

Dorigo y Gambardella (1997) introducen el Sistema de Colonia de Hormigas (ACS por sus siglas en inglés para Ant Colony System) para solucionar instancias TSP. En este trabajo muestran que el ACS supera a otros algoritmos inspirados en la naturaleza, como Recocido Simulado, Algoritmos Genéticos y Programación Evolutiva (compara con resultados de Fogel, 1993, entre otros). Además, prueban una versión aumentada del ACS con un procedimiento de búsqueda local 3-opt, arrojando resultados satisfactorios en el TSP. Stuetzle y Hoos (1997) exponen el Sistema de Hormigas Max-Min, una extensión que mejora la estructura básica del ACS, probada en TSP de hasta 783 ciudades; igualmente, muestran que el método propuesto puede ser mejorado con un procedimiento de búsqueda local tipo $k$-opt, orientando dicha búsqueda hacia regiones prometedoras mediante la generación de buenos recorridos iniciales.

Larrañaga et al. (1999) hacen una revisión de los diferentes intentos para resolver el TSP con Algoritmos Genéticos. Presentan operadores de cruce y de mutación con diferentes representaciones, entre ellas: binaria, ordinal, adyacencia y matricial. Los autores también comparan la efectividad de los operadores, empleando un algoritmo genético apoyado en principios del GENITOR (WHITLEY; STARKWEATHER; FUQUAY, 1989), probándolo hasta para 48 ciudades.

Tabla 3. Clasificaciones de TSP, métodos y campos de inspiración.

\begin{tabular}{|c|c|c|c|c|}
\hline Estudio & Clase & Método(s) basado(s) en & Campo de inspiración & $\begin{array}{l}\text { Tamaño } \\
\text { máximo } \\
\text { probado }\end{array}$ \\
\hline Held y Karp (1971) & TSP & Relajación lagrangiana & Ramificación y acotamiento & 64 \\
\hline Cerný (1985) & TSP & Recocido simulado, Método de Montecarlo & Termodinámica & 200 \\
\hline $\begin{array}{l}\text { Angéniol, De La Croix } \\
\text { y Le Texier (1988) }\end{array}$ & TSP & $\begin{array}{c}\text { Mapas auto-organizados de Kohonen, Red } \\
\text { neuronal }\end{array}$ & Biofísica del cerebro & 1000 \\
\hline Laporte (1992) & TSP & $\mathrm{N} / \mathrm{A}$ & $\mathrm{N} / \mathrm{A}$ & $\mathrm{N} / \mathrm{A}$ \\
\hline Reinelt (1991) & TSP & $\mathrm{N} / \mathrm{A}$ & $\mathrm{N} / \mathrm{A}$ & $\mathrm{N} / \mathrm{A}$ \\
\hline Bentley (1992) & TSP & $\begin{array}{l}\text { Inserción (vecino más cercano, ...), basados en } \\
\text { árboles y búsqueda local (2-opt, } 2 \mathrm{H} \text {-opt, 3-opt) }\end{array}$ & $\begin{array}{c}\text { Estrategias rápidas de inicialización } \\
\text { y búsqueda de vecindades }\end{array}$ & 1000000 \\
\hline Fogel (1993) & TSP & Programación evolutiva & Evolución biológica & 1000 \\
\hline $\begin{array}{l}\text { Dorigo y Gambardella } \\
\text { (1997) }\end{array}$ & TSP & $\begin{array}{l}\text { Sistema de Colonia de Hormigas, 3-opt } \\
\text { (Búsqueda local) }\end{array}$ & $\begin{array}{c}\text { Comportamiento de hormigas reales } \\
\text { y búsqueda de vecindades }\end{array}$ & 1577 \\
\hline $\begin{array}{l}\text { Stuetzle y Hoos } \\
\text { (1997) }\end{array}$ & TSP & $\begin{array}{l}\text { Sistema de Hormigas Max-Min, 2-opt, 3-opt } \\
\text { (Búsqueda local) }\end{array}$ & $\begin{array}{c}\text { Comportamiento de hormigas reales } \\
\text { y búsqueda de vecindades }\end{array}$ & 783 \\
\hline $\begin{array}{l}\text { Larrañaga et al. } \\
\qquad \text { (1999) }\end{array}$ & TSP & Algoritmos genéticos. GENITOR & $\begin{array}{l}\text { Evolución biológica y su base } \\
\text { genético-molecular }\end{array}$ & 48 \\
\hline Bektas (2006) & m-TSP & $\mathrm{N} / \mathrm{A}$ & $\mathrm{N} / \mathrm{A}$ & $\mathrm{N} / \mathrm{A}$ \\
\hline $\begin{array}{l}\text { Carter y Ragsdale } \\
\text { (2006) }\end{array}$ & m-TSP & Algoritmos genéticos & $\begin{array}{l}\text { Evolución biológica y su base } \\
\text { genético-molecular }\end{array}$ & 150 \\
\hline $\begin{array}{l}\text { Snyder y Daskin } \\
(2006)\end{array}$ & GTSP & Algoritmos genéticos y 2-opt (Búsqueda local) & $\begin{array}{l}\text { Evolución biológica y su base } \\
\text { genético-molecular; búsqueda de } \\
\text { vecindades }\end{array}$ & 442 \\
\hline Campbell (2006) & PTSP & $\begin{array}{c}\text { Segregación de clientes y resolución del } \\
\text { problema reducido }\end{array}$ & Fragmentación urbana & 1000 \\
\hline Nguyen et al. (2007) & TSP & Algoritmos genéticos, LK (Búsqueda local) & $\begin{array}{l}\text { Evolución biológica y su base } \\
\text { genético-molecular, búsqueda de } \\
\text { vecindades }\end{array}$ & 1904711 \\
\hline Liu (2007) & PTSP & $\begin{array}{c}\text { Búsqueda dispersa hibrido. Vecino más cercano, } \\
\text { Búsqueda local (aceptación por umbrales), } \\
\text { operador de recombinación }\end{array}$ & $\begin{array}{l}\text { Métodos evolutivos (Estrategias } \\
\text { sistemáticas para combinar reglas de } \\
\text { decisión), búsqueda de vecindades }\end{array}$ & 100 \\
\hline $\begin{array}{l}\text { Carrabs, Cordeau y } \\
\text { Laporte (2007) }\end{array}$ & TSPPDL & $\begin{array}{c}\text { Búsqueda de entorno variable y operadores de } \\
\text { búsqueda local }\end{array}$ & $\begin{array}{c}\text { Cambios sistemáticos de entorno } \\
\text { dentro de una búsqueda de } \\
\text { vecindades }\end{array}$ & 721 \\
\hline $\begin{array}{l}\text { Ohlmann y Thomas } \\
\text { (2007) }\end{array}$ & TSPTW & $\begin{array}{c}\text { Variante del Recocido simulado (Compressed } \\
\text { annealing). }\end{array}$ & $\begin{array}{l}\text { Recocido del acero, Métodos de } \\
\text { penalización }\end{array}$ & 200 \\
\hline Duan y Yu (2007) & TSP & $\begin{array}{c}\text { Sistema de Colonia de Hormigas y Algoritmos } \\
\text { meméticos }\end{array}$ & $\begin{array}{l}\text { Comportamiento de hormigas } \\
\text { reales y Evolución cultural de los } \\
\text { individuos }\end{array}$ & 51 \\
\hline $\begin{array}{l}\text { Savla, Frazzoli y Bullo } \\
\text { (2008) }\end{array}$ & DTSP & $\begin{array}{l}\text { Alternating Algorithm. Algoritmo de } \\
\text { aproximación de factor constante }\end{array}$ & $\begin{array}{l}\text { Solución óptima del TSP simétrico. } \\
\text { Algoritmos de aproximación }\end{array}$ & N/A \\
\hline
\end{tabular}

Estudio (publicación), clase, tipo de método, campo de inspiración y tamaño (en número de ciudades) de las instancias probadas en estudios sobre TSP. La sigla N/A indica que no aplica, por tratarse de estudios de tipo revisión. Fuente: Pérez (2011). 


\section{2. ¿De qué tratan las diez publicaciones más citadas en el periodo 2006-2010, que abordan el TSP como eje central?}

Bektas (2006) presenta una revisión del $m$-TSP (Multiple Traveling Salesman Problem), el cual consta de $m>1$ vendedores para visitar $n>m$ localidades una sola vez, minimizando el recorrido o coste total; también trata la definición, formulaciones, aplicaciones y métodos de resolución de esta variante del TSP. Carter y Ragsdale (2006) tratan el $m$-TSP mediante algoritmos genéticos, probando un nuevo cromosoma no estándar ("Two-part chromosome"). Comparando el desempeño computacional de su propuesta con enfoques convencionales de estudios previos con cromosomas estándar, encuentran en algunos casos mejores soluciones para el $m$-TSP, con instancias de hasta 150 ciudades.

Snyder y Daskin (2006) tratan el problema del vendedor viajero generalizado (GTSP), en el que existen clusters o grupos predefinidos y el viajero debe visitar al menos un nodo en cada cluster, minimizando el costo total del viaje. Presentan una heurística que combina algoritmos genéticos de llaves aleatorias (random-key) con una búsqueda local. Para 41 problemas de prueba con distancias simétricas y hasta 442 nodos, encuentran el valor óptimo en la mayoría de los casos, mientras que en los demás las soluciones estuvieron dentro del $1 \%$ del óptimo, con una eficiencia computacional dentro de los 10 segundos ( $\mathrm{C}_{++}$, en Pentium IV de $3.2 \mathrm{GHz}$ ). Concluyen que la heurística propuesta es competitiva en calidad y eficiencia, en comparación con otros métodos de solución (cuatro heurísticas y un algoritmo exacto con instancias del TSPLIB).

Campbell (2006) se orienta hacia el PTSP (problema del vendedor viajero probabilístico); en éste caso, solo un subconjunto de clientes potenciales deben ser visitados, siendo el número de clientes a visitar una variable aleatoria. Ante la dificultad de resolver esta variante para instancias de tamaños propios de casos reales, una de las alternativas es asignar clientes a regiones y resolver para una instancia más reducida. Es así que el interrogante que abordaron los autores fue la forma de dividir a los clientes y la escala necesaria para representar razonablemente la función objetivo. El estudio ofrece resultados computacionales en problemas de hasta 1.000 ciudades, donde las probabilidades de visitar a los clientes son bajas y el tiempo computacional disponible es reducido; es decir, la eficiencia computacional juega un papel preponderante. Campbell (2006) manifiesta que la agregación de clientes en regiones puede proporcionar estimaciones cercanas al objetivo de manera rápida $\mathrm{y}$, resolver el primer problema (reducido), puede conducir a soluciones satisfactorias a menor costo.
Nguyen et al. (2007) implementan un algoritmo genético con un método de búsqueda local basado la heurística LK (LIN; KERNIGHAN, 1973) para encontrar soluciones de alta calidad en instancias TSP de gran escala. Los resultados experimentales muestran que el método propuesto por los autores funcionó con mayor eficacia y eficiencia que el LKH (HELSGAUN, 1998), uno de los más destacados en la resolución del TSP. Además, prueban el método para encontrar una mejor solución para una instancia de TSPLIB que presenta 1.904.711 ciudades. Liu (2007) enfoca el PTSP desarrollando un algoritmo híbrido de búsqueda dispersa que incorpora, entre otros, la regla del Vecino más Cercano, aceptación por umbrales y un operador de recombinación (Edge Recombination). Destaca la potencialidad de la propuesta para resolver problemas de gran tamaño, mostrando que la incorporación del umbral de aceptación dentro de la búsqueda dispersa favorece la eficiencia del procedimiento manteniendo la calidad de la solución.

Carrabs, Cordeau y Laporte (2007) enfocan el TSPPDL, Problema del vendedor viajero con recogida y entrega de mercancía y restricciones de carga y descarga LIFO (últimas en entrar, primeras en salir). Para referir a este problema es necesario describir el "Traveling Salesman Problem with Pickup and Delivery" (TSPPD), que consiste en determinar el recorrido de longitud mínima que se requiere para que un solo vehiculo, con capacidad de carga limitada, satisfaga los requerimientos de los clientes, a los cuales debe recogerles la mercancía en un determinado sitio de origen, para su posterior entrega en el sitio de destino; la política óptima debe satisfacer las restricciones de capacidad de carga limitada del vehículo y las de precedencia entre origen y destino. En el TSPPDL se agregan restricciones de precedencia que incorporan la política LIFO al cargar y extraer la mercancía. Proponen tres nuevos operadores de búsqueda local, los cuales se incorporan a la heurística de búsqueda de entorno variable, probando el rendimiento del algoritmo con instancias adaptadas de TSPLIB, obteniendo resultados satisfactorios en eficiencia y eficacia en comparación con el método de prueba tipo búsqueda entorno variable descendente (VND) de Cassani y Righini (2004).

Ohlmann y Thomas (2007) orientan sus esfuerzos hacia el TSPTW (Travelling Salesman Problem with Windows Time), en el cual deben considerarse restricciones de tiempo para las visitas a los clientes, llevando a que cada ciudad deba ser visitada en un tiempo determinado, no permitiéndose llegar después de dicho tiempo; igualmente, si el viajero llega antes, debe esperar que se cumpla el tiempo preestablecido. Usan una variante del Recocido simulado, denominada Compressed-Annealing, la cual relaja las restricciones de ventanas de tiempo e incorpora un método de 
penalización de variable asociado al concepto de presión. Hasta 200 ciudades, el método resulta satisfactorio en la mayoría de los casos. Duan y Yu (2007) resaltan la robustez de la optimización bajo Colonia de Hormigas y su facilidad para integrarse con otros métodos. Los autores combinan ACS con Algoritmos meméticos. Al final proponen un nuevo enfoque y lo prueban para un TSP de 51 ciudades, mostrando eficacia y viabilidad práctica.

Savla, Frazzoli y Bullo (2008) hacen una aproximación del TSP a la robótica, enfocados en el enrutamiento de un vehículo tipo Dubins, denominándola DTSP. Destacan que el TSP y sus variaciones continúan atrayendo esfuerzos de diferentes disciplinas, como las matemáticas, las ciencias de la computación e investigación de operaciones. El DTSP es similar al TSP, puesto que el vehículo debe visitar una sola vez una serie de puntos y regresar al origen, pero con la característica de que deben considerarse restricciones asociadas a las trayectorias de curvatura que sigue el vehículo, delimitadas en el plano. Proponen, entre otros, un algoritmo llamado "Alternating Algorithm" que incorporan dentro de la categoría de "algoritmo de aproximación de factor constante". Mayor profundización en este tipo de procedimientos puede encontrarse en Vazirani (2001).

\section{3. ¿Qué clases de TSP han sido abordados} en los estudios más citados sobre el tema, través de la historia y entre 2006-2010?; ¿Qué métodos de resolución han sido empleados en los estudios más citados sobre el TSP? y ¿Qué campos han inspirado a los métodos de resolución empleados en los estudios más citados sobre el TSP?

Los diez estudios mejor posicionados sobre el TSP a través de la historia, publicados entre 1971 y 1999, abordaron el TSP original (Tabla 3). Respecto a 2006-2010, dos de los diez documentos más citados abordaron el TSP original, en tanto que los demás enfocaron las siguientes variaciones: PTSP (dos estudios), m-TSP (dos estudios), TSPDL (un estudio), TSPTW (un estudio) y DTSP (un estudio) y GTSP (un estudio). Los métodos empleados en los trabajos objeto de este análisis pueden verse en la Tabla 3 (respuestas a las preguntas 4 y 5); los campos de inspiración más destacados fueron: evolución biológica y su base genético-molecular, comportamiento de hormigas reales, búsqueda de vecindades, estrategias sistemáticas para combinar reglas de decisión, y cambios sistemáticos de entorno. El tamaño máximo de las instancias probadas en los estudios abordados osciló entre 48 y 1'000.000 de ciudades, según se expone en la Tabla 3.

\section{Discusión de resultados}

\subsection{Tendencias en el espacio literario relevante abordado}

El TSP ocupa un papel crucial en la investigación de operaciones, tanto a nivel teórico como aplicado, siendo dicho problema reconocido por su complejidad de resolución, sencilla formulación y prueba de validación inicial de nuevos avances algorítmicos en la optimización combinatoria (ANGÉNIOL; DE LA CROIX; LE TEXIER, 1988; LAPORTE, 1992; BOESE; KAHNG; MUDDU, 1994; JOHNSON; McGEOCH, 1997; MARTI, 2003; DONG; GUO; TICKLE, 2012). Probar la efectividad de los métodos emergentes en el TSP posibilita luego trascender hacia otros problemas, como ocurrió con la relajación lagrangiana, con el branch and bound (ÁLVAREZ; CORBERÁN; TAMARIT, 1985) y con el modelo inicial de Colonia de Hormigas (DORIGO; GAMBARDELLA, 1995).

Desde 1954 se ha incrementado la efectividad de los métodos desarrollados para resolver problemas TSP cada vez de mayor tamaño. Por ejemplo, Dantzig, Fulkerson y Johnson (1954) resolvieron para 49 ciudades, Held y Karp (1971) para 64 ciudades, Padberg y Rinaldi (1987) aportaron respuesta a una instancia de 2.392 ciudades y en el 2004 ya se conocía respuesta para 24.978 ciudades (APPLEGATE et al., 2006). El TPS de mayor tamaño resuelto hasta la fecha ha sido para una instancia de 85.900 ciudades (APPLEGATE et al., 2006), denominada pla 85.900 en el dominio público TSPLIB. En dicha librería también se dispone de problemas de mayores tamaños, con la mejor solución encontrada.

Los principales métodos para aportar respuestas al TSP pueden dividirse en: algoritmos heurísticos, de enumeración implícita y metaheurísticas. Entre las heurísticas clásicas para el TSP están las de construcción son: Vecino más Cercano, de inserción (más lejana, más cercana); las de mejoramiento son otra categoría: $k$-opt, LK y LKH; respecto a métodos de enumeración implícita, están los algoritmos tipo Branch and bound. Entre las metaheurísticas de los estudios más posicionados para el TSP están: Algoritmos Genéticos, Sistema Colonia de Hormigas, Búsqueda Dispersa, Búsqueda de Entorno Variable y Recocido Simulado.

Con base en el estudio de Savla, Frazzoli y Bullo (2008) donde se menciona el término "algoritmo aproximado de factor constante", se destaca que algunos estudios no distinguen entre "algoritmos aproximados" y los "algoritmos heurísticos", mientras que otros sí lo hacen. Partiendo de los que diferencian dicha agrupación, se menciona que una heurística, a pesar de ser eficiente desde el punto de vista 
práctico, no tiene una medida teórica de la calidad del algoritmo ni del tiempo de ejecución, en tanto que los aproximados llegan, en un tiempo polinomial, a una respuesta cercana a la óptima, pero sabiendo qué tan lejos de ella se encuentran (MONTOYA; PATERNINA; FREIN, 2002; VERSCHAE, 2008; SCHAEFFER, 2009). Entre los que no diferencian sobre la mencionada clasificación, están Ríos y Bard (2000), Vélez y Montoya (2007) y Quevedo y Ríos (2010).

A medida que se avanza en el desarrollo de nuevos métodos de resolución para el TSP y, a su vez, se resuelven instancias cada vez más complejas, el probar la efectividad de dichos métodos a través de instancias de grandes tamaños no parece ser un requisito para los trabajos futuros, puesto que en los estudios analizados se encuentran experimentos válidos empleando instancias de tamaño máximo, no necesariamente crecientes a través del tiempo: 1.577 ciudades (DORIGO; GAMBARDELLA, 1997), 48 ciudades (LARRAÑAGA et al., 1999), 51 ciudades (DUAN; YU, 2007), 100 ciudades (CARRABS; CORDEAU; LAPORTE, 2007) y 1.904 .711 ciudades (NGUYEN et al. 2007). Respecto a dichas pruebas computacionales, se destaca el aporte del TSPLIB (REINELT, 1991), prevaleciendo como una fuente de información de amplio uso en los estudios mejor posicionados, vital para realizar comparaciones entre los métodos existentes, facilitar validaciones de las nuevas propuestas y posibilitar el reconocimiento de las contribuciones más destacadas.

En vista de los avances en el tema, el TSP aún prevalece como un importante campo de conocimiento, puesto que además del caso original, que, como se ha evidenciado, aún constituye un atractivo para la comunidad académica, se destacan también en los estudios mejor posicionados seis de sus variantes: m-TSP, PTSP, TSPPDL, TSPTW, GTSP y DTSP. Es así que desarrollar nuevos métodos para aportar respuestas satisfactorias al TSP contribuye no solo al progreso académico, sino también a un significativo avance en el desempeño de las organizaciones industriales y de servicios, cuyo modo de operación se relaciona directamente o en parte con la estructura de este problema (GONZÁLEZ; RÍOS, 1999).

En los estudios más citados a través de la historia se nota el enfoque hacia el TSP original, pero luego, observando 2006-2010, ya previamente validados los métodos existentes en este referente de prueba, comienza a notarse el enfoque hacia las variantes del TSP, así como una mayor tendencia hacia la combinación de las fortalezas entre diferentes métodos, dando lugar a híbridos de las metaheurísticas. Entre las combinaciones de métodos empleados en los estudios más citados, se destacan: Sistema de Colonia de Hormigas con k-opt (DORIGO; GAMBARDELLA, 1997; STUETZLE; HOOS, 1997) y con Algoritmos meméticos (DUAN; YU, 2007); también los Algoritmos genéticos con LK (NGUYEN et al., 2007) y con k-opt (SNYDER; DASKIN, 2006), así como la combinación de la metaheurística de búsqueda dispersa con la búsqueda local (aceptación por umbrales) (LIU, 2007). A pesar de la antigüedad de algunos optimizadores locales, éstos aún prevalecen en los mecanismos académicos para aportar al TSP y sus variantes, empleándolos para combinar enfoques de búsqueda global con perspectivas locales, notándose el 2-opt como uno de los más usados.

Además de ser fundamental avanzar, en trabajos futuros, alrededor del conocimiento actual sobre el TSP, siguiendo la tendencia de generar métodos híbridos, existe una opción adicional para potenciar la generación de nuevo conocimiento en el tema. Esta alternativa resulta de analizar el origen de los métodos existentes, los cuales, como se ilustra en la Figura 1, parten de la introducción de nuevos campos de inspiración, que incorporen otras perspectivas, y que luego den lugar al desarrollo de nuevos métodos. Ello ocurrió, por ejemplo, con métodos hoy posicionados, como ACO (hormigas reales), redes neuronales (biofísica del cerebro), algoritmos genéticos (evolución biológica y su base genético-molecular) y recocido simulado (termodinámica), entre otros. Estos métodos fueron probados inicialmente en el TSP, luego en sus variantes, y finalmente trascendieron hacia diversos campos de aplicación y hacia la generación de híbridos. Aunque resulte más compleja, dicha oportunidad de aportar al TSP puede tener un mayor impacto en el avance general de la optimización combinatoria.

\subsection{Tendencias del espacio literario relevante en estudios recientes (2012)}

Con el fin de explorar las anteriores tendencias en estudios recientes, el 02 de abril de 2012 se ejecutó el algoritmo de búsqueda expuesto en la Figura 2, en la base de datos Scopus, encontrándose 20 estudios, delimitados a artículos de revistas y artículos de conferencias en Ingenierías, Ciencias de la Computación y Ciencias de la Decisión.

Al estudiar dichos trabajos, se notó que prevalecen en la actualidad tanto los enfoques hacia el TSP como problema de prueba, propio de lo encontrado en los diez artículos más citados a través de la historia, como también hacia sus variantes (más citados entre 2006-2010). De esta manera, 11 de los 20 documentos localizados tratan variantes conocidas de este problema o proponen otras nuevas, según se muestra en la Tabla 4.

En estos estudios subyace la adaptación o mejoramiento de métodos previamente probados en el TSP original, o de profundización en la estructura de este problema, o nuevas variantes de TSP con particularidades propias de otros campos de aplicación. 


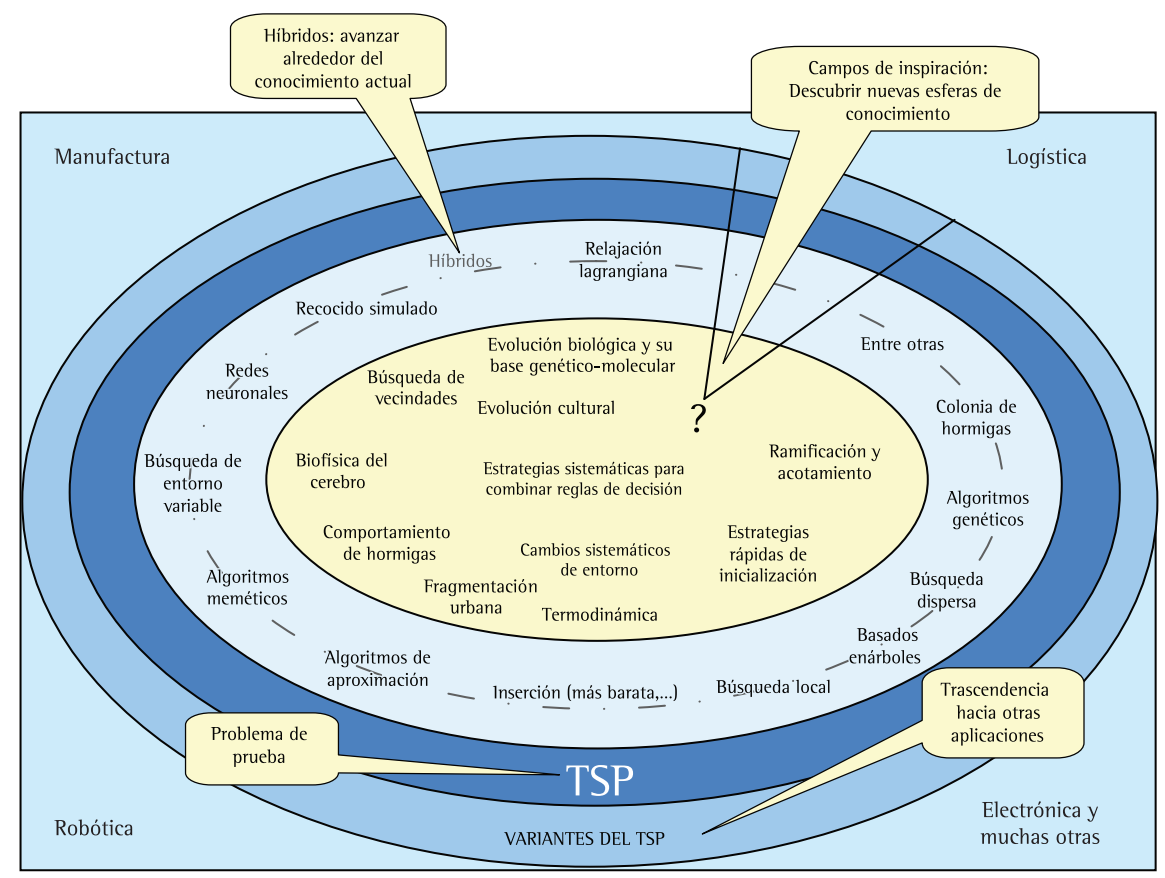

Figura 1. llustración de la tendencia para avanzar alrededor del conocimiento actual sobre el TSP y para mover las puntas de conocimiento hacia otras esferas.

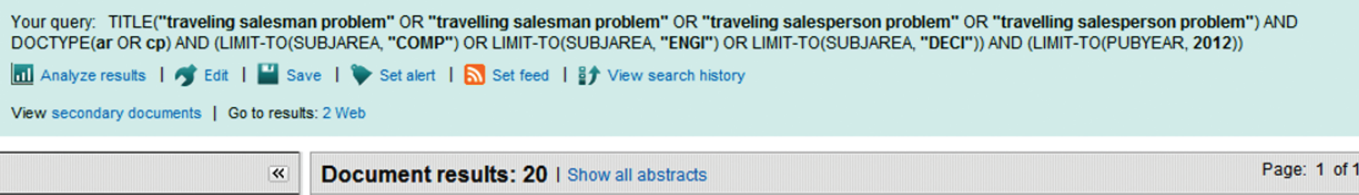

Figura 2. Algoritmo de búsqueda de estudios del 2012 sobre el TSP.

Los nueve documentos restantes tratan propiamente el TSP (simétrico o asimétrico), algunos analizando, por ejemplo, el efecto de diversos factores sobre la asimetría del problema (RODRÍGUEZ; RUIZ, 2012), o estudiándolo bajo una estructura de red Bayesiana (CHEN, 2012) o profundizando teóricamente en su resolución a través de ACO. Además, en cinco de los nueve documentos se nota una mayor inclinación a emplear el TSP como prueba para validar nuevos métodos, resultantes de hibridación de metaheurísticas: ACO con memoria (WANG; ZHAO; ZHOU, 2012); Búsqueda tabú con algoritmos de peces artificiales (CHEN et al., 2012b); PSO (Particle Swarm Optimization) con Sistemas inmunes artificiales (CHEN et al., 2012c); ACO, algoritmos genéticos y recocido simulado (CHEN et al., 2012d); PSO y recocido simulado (WU; WU; LIU, 2012); se destaca que Erdogan et al. (2012) combina Búsqueda tabú con programación dinámica, pero para una variante del TSP.

Así mismo, al explorar otros estudios mediante una búsqueda no sistemática, puede verse también la misma tendencia de combinación de metaheurísticas y de prevalencia del TSP y de sus variantes. Algunas evidencias de ello son Marinakis, Marinaki y Dounias (2011) quienes aplicaron, de forma híbrida, la metaheurística HBMO (Honey bees mating optimization) para resolver el TSP, integrándola con "Multiple Phase Neighborhood Search-Greedy Randomized Adaptive" y "Expanding Neighborhood Search Strategy"; Dong, Guo y Tickle (2012), ofrecen una nueva extensión de ACO para el TSP, que denominaron Cooperative Genetic Ant System (CGAS) que combina Algoritmos genéticos y ACO; Chen y Chien (2011) también siguieron esta tendencia, exponiendo un híbrido que al llamaron Genetic Simulated Annealing Ant Colony System With Particle Swarm Optimization para resolver el TSP; Marinakis y Marinaki (2010) presentaron un algoritmo un hibrido de Particle Swarm Optimization (PSO), Greedy Randomized Adaptive Search Procedure (GRASP) y Expanding Neighborhood Search (ENS) Strategy, para aportar respuestas al PTSP. 
Tabla 4. Clasificaciones de TSP en algunos estudios publicados en el 2012.

\begin{tabular}{|c|c|}
\hline Estudio & Clase de TSP \\
\hline Glomvik et al. (2012) & TSP with Draft Limits (TSPDL) \\
\hline Mladenović et al. (2012) & $\begin{array}{l}\text { One-commodity Pickup-and- } \\
\text { Delivery TSP (1-PDTSP) }\end{array}$ \\
\hline Karapetyan y Gutin (2012) & GTSP \\
\hline Erdogan et al. (2012) & $\begin{array}{l}\text { TSP with Pickups, Deliveries, and } \\
\text { Handling Costs (TSPPD-H) }\end{array}$ \\
\hline $\begin{array}{l}\text { Casazza, Ceselli y Nunkesser, } \\
\text { (2012) }\end{array}$ & $\begin{array}{l}\text { Double TSP with Multiple Stacks } \\
\text { (DTSPMS) }\end{array}$ \\
\hline Cheng et al. (2012a) & Bi-objective TSP (bTSPS) \\
\hline $\begin{array}{l}\text { Weyland, Montemanni y } \\
\text { Gambardella (2012) }\end{array}$ & $\begin{array}{l}\text { Probabilistic TSP with Deadlines } \\
(P T S P D)\end{array}$ \\
\hline $\begin{array}{l}\text { Vansteenwegen, Souffriau y } \\
\text { Sörensen (2012) }\end{array}$ & TSP with Hotel Selection (TSPHS) \\
\hline Su, Cao y Zuo (2012) & TSP on a cubrid (CuboidTSP) \\
\hline Ny, Feron y Frazzoli (2012) & DTSP \\
\hline $\begin{array}{l}\text { Weyland, Montemanni y } \\
\text { Gambardella (2012) }\end{array}$ & $\begin{array}{l}\text { Probabilistic TSP with Deadlines } \\
(P T S P D)\end{array}$ \\
\hline
\end{tabular}

\section{Conclusiones}

Como resultado de la introducción en las publicaciones sobre el TSP como eje central, más citadas a través de la historia y entre 2006-2010, se aportó evidencia sobre el contenido de los estudios, la vigencia del tema, la clasificación de los TSP abordados, los métodos y los campos de inspiración. Esto, de modo general, permitió deducir que para avanzar desde el conocimiento actual se tiende hacia la hibridación de los métodos existentes, en tanto que para avanzar hacia esferas de conocimiento desconocidas es preciso introducir nuevos campos de inspiración que luego den origen a noveles métodos heurísticos, posibilitando un mayor avance en la optimización combinatoria. Se evidenció una tendencia a probar los nuevos métodos que se van generando, sean resultado de hibridación o nuevos campos de inspiración, en el TSP original como medio de validación; luego de ello, se nota una corriente de aplicaciones hacia sus variantes ya existentes o a proponer otras. Esto último debido a la identificación de particularidades en problemas reales, relacionados de algún modo con el TSP original, que ameritan adaptaciones para ser representados razonablemente bajo la estructura general de este problema, para luego ser abordados.

Las preguntas de investigación saldadas en este artículo no deberían faltar en defensas de tesis de Pregrado o Posgrado en el tema. De ahí la importancia de este trabajo para estudiantes e investigadores que deseen realizar contribuciones para el avance en dicho campo de investigación.

\section{Referencias}

ÁlVAREZ, R.; CORBERÁN, A.; TAMARIT, J. La combinatoria poliédrica y el problema del viajante. Aplicación al caso de ciento tres ciudades Españolas. Qüestió, v. 9, n. 3, p. 199-213, 1985.

ANGÉNIOL, B.; DE LA CROIX, V.; LE TEXIER, J. Self-organizing feature maps and the travelling salesman problem. Neural Networks, v. 1, n. 4, p. 289-293, 1988. http:// dx.doi.org/10.1016/0893-6080(88)90002-0

APPLEGATE, D. et al. The traveling salesman problem: a computational study. New Jersey: Princeton University Press, 2006. p. 1-593.

BEKTAS, T. The multiple traveling salesman problem: An overview of formulations and solution procederes. Omega, v. 34, n. 3, p. 209-219, 2006. http://dx.doi. org/10.1016/j.omega.2004.10.004

BENTLEY, J. Fast algorithms for geometric traveling salesman problems. ORSA journal on computing, v. 4, n. 4, p. 387-411, 1992.

BOESE, K.; KAHNG, A.; MUDDU, S. A new Adaptive Multi-Start Technique for Combinatorial Global Optimization. Operations Research Center, v. 16, p. 101-113, 1994. http://dx.doi.org/10.1016/0167-6377(94)90065-5

CAMPBELL, A. Aggregation for the probabilistic traveling salesman problem. Computers and Operations Research, v. 33, n. 9, p. 2703-2724, 2006. http://dx.doi. org/10.1016/j.cor.2005.02.024

CARRABS, F.; CORDEAU, J.; LAPORTE, G. Variable neighborhood search for the pickup and delivery traveling salesman problem with LIFO loading. INFORMS Journal on Computing, v. 19, n. 4, p. 618-632, 2007. http://dx.doi.org/10.1287/ijoc. 1060.0202

CARTER, A.; RAGSDALE, C. A new approach to solving the multiple traveling salesperson problem using genetic algorithms. European Journal of Operational Research, v. 175 , n. 1, p. 246-257, 2006. http://dx.doi. org/10.1016/j.ejor.2005.04.027

CASAZZA, M.; CESELLl, A.; NUNKESSER, M. Efficient algorithms for the double traveling salesman problem with multiple stacks. Computers and Operations Research, v. 39, n. 5, p. 1044-1053, 2012. http://dx.doi. org/10.1016/j.cor.2011.06.008

CASSANI, L.; RIGHINI, G. Heuristic algorithms for the TSP with rear-loading. In: ANNUAL CONFERENCE OF THE ITALIAN OPERATIONAL RESEARCH SOCIETY (AIRO XXXV), 35., 2004, Lecce, 1taly. Proceedings... Lecce, 2004.

CERNÝ, V. Thermodynamical approach to the traveling salesman problem: An efficient simulation algorithm. Journal of Optimization Theory and Applications, v. 45, n. 1, p. 41-51, 1985. http://dx.doi.org/10.1007/ BF00940812

CHEN S.; CHIEN, C. Solving the traveling salesman problem based on the genetic simulated annealing ant colony system with particle swarm optimization techniques. Expert Systems with Applications, v. 38, n. 12, p. 14439-14450, 2011. http://dx.doi.org/10.1016/j. eswa.2011.04.163

CHEN, N. An ant colony optimization and Bayesian network structure application for the asymmetric traveling salesman problem. Lecture Notes in Computer Science (including subseries Lecture Notes in Artificial Intelligence and Lecture Notes in Bioinformatics), 7198 LNAl (Part 3), p. 74-78, 2012.

CHEN, X. et al. Chaotic taboo fish algorithm for traveling salesman problem. Lecture Notes in Electrical Engineering, 143 LNEE, v. 1, p. 697-704, 2012b.

CHEN, X. et al. Chaotic immune PSO algorithm for traveling salesman problem. Lecture Notes in Electrical Engineering, 143 LNEE, v.1, p. 689-695, 2012c. 
CHEN, X. et al. A hybrid algorithm to solve traveling salesman problem. Lecture Notes in Electrical Engineering, 139 LNEE, p. 99-105, 2012d.

CHENG, J. et al. Multi-objective ant colony optimization based on decomposition for bi-objective traveling salesman problems. Soft Computing, v. 16, n. 4, p. 597-614, 2012a. http://dx.doi.org/10.1007/s00500011-0759-3

CODINA, L. El mayor navegador científico de la Web. El profesional de la información, v. 14, n. 1, p. 44-49, 2005. http://dx.doi.org/10.3145/epi.2005.feb.07

DANTZIG, G.; FULKERSON, R.; JOHNSON, S. Solution of a large-scale traveling-salesman problem. Journal of the operations research society of America, v. 2, n, 4, p. 393-410, 1954.

DAVIS, D. et al. Changing physician performance: A systematic review of the effect of continuing medical education strategies. Journal of the American Medical Association, v. 274, n. 9, p. 700-705, 1995. http://dx.doi. $\operatorname{org} / 10.1001 /$ jama.1995.03530090032018

DONG, G.; GUO, W.; TICKLE, K. Solving the traveling salesman problem using cooperative genetic ant systems. Expert Systems with Applications, v. 39, n. 5, p. 5006-5011, 2012. http://dx.doi.org/10.1016/j. eswa.2011.10.012

DORIGO, M.; GAMBARDELLA, L. Ant colony system: A cooperative learning approach to the traveling salesman problem. IEEE Transactions on Evolutionary Computation, v. 1, n. 1, p. 53-66, 1997. http://dx.doi. org/10.1109/4235.585892

DORIGO, M.; GAMBARDELLA, M. Ant-q: A reinforcement learning approach to the traveling salesman problem. In: INTERNATIONAL CONFERENCE ON MACHINE LEARNING, 12., 1995, Tahoe City. Proceedings... Tahoe City, 1995. p. 252-260.

DUAN, H.; YU, X. Hybrid ant colony optimization using memetic algorithm for traveling salesman problem. IEEE ADPRL 2007, art. n. 4220819, p. 92-95, 2007.

ERDOGAN, G. et al. Metaheuristics for the traveling salesman problem with pickups, deliveries and handling costs. Computers and Operations Research, v. 39, n. 5, p. 1074-1086, 2012. http://dx.doi.org/10.1016/j. cor.2011.07.013

FOGEL, D. Applying evolutionary programming to selected traveling salesman problems. Cybernetics and Systems, v. 24, n. 1, p. 27-36, 1993. http://dx.doi. org/10.1080/01969729308961697

GLOMVIK, J. et al. The Traveling salesman problem with draft limits. Computers and Operations Research, v. 39, n. 9, p. 2161-2167, 2012. http://dx.doi.org/10.1016/j. cor.2011.10.025

GONZÁLEZ, J.; RíOS, R. Investigación de operaciones en acción: Aplicación del TSP en problemas de manufactura y logística. Ingenierías, v. 2, n. 4, p. 18-23, 1999.

HELD, M.; KARP, R. The traveling-salesman problem and minimum spanning trees: Part 11. Mathematical Programming, v. 1, n. 1, p. 6-25, 1971. http://dx.doi. org/10.1007/BF01584070

HELSGAUN, K. An effective implementation of the Lin-Kernighan Traveling Salesman Heuristic. Datalogiske Skrifter (Writings on Computer Science), n. 81, p. 1-71, 1998.

JOHNSON, D.; McGEOCH, L. The Traveling Salesman Problem: A Case Study in Local Optimization. In: AARTS, E.; LENSTRA, J. (Eds.). Local Search in Combinatorial
Optimization. Chichester: Wiley, 1997. chapt. 8, p. 215-310.

KARAPETYAN, D.; GUTIN, G. Efficient local search algorithms for known and new neighborhoods for the generalized traveling salesman problema. European Journal of Operational Research, v. 219, n. 2, p. 234-251, 2012. http://dx.doi.org/10.1016/j.ejor.2012.01.011

KITCHENHAM, B. et al. Systematic literature reviews in software engineering - A systematic literature review. Information and Software Technology, v. 51, n. 1, p. 7-15, 2009. http://dx.doi.org/10.1016/j.infsof.2008.09.009

LAPORTE, G. The traveling salesman problem: An overview of exact and approximate algorithms. European Journal of Operational Research, v. 59, n. 2, p. 231-247, 1992. http://dx.doi.org/10.1016/0377-2217(92)90138-Y

LARRAÑAGA, P. et al. Genetic algorithms for the travelling salesman problem: A review of representations and operators. Artificial Intelligence Review, v. 13, n. 2, p. 129170, 1999. http://dx.doi.org/10.1023/A:1006529012972

LIN, S.; KERNIGHAN, B. An effective heuristic algorithm for the Traveling-Salesman Problem. Operations Research, v. 21 , n. 2 , p. $498-516,1973$. http://dx.doi.org/10.1287/ opre.21.2.498

LIU, Y. A hybrid scatter search for the probabilistic traveling salesman problem. Computers and Operations Research, v. 34, n. 10, p. 2949-2963, 2007. http://dx.doi. org/10.1016/j.cor.2005.11.008

MARINAKIS, Y.; MARINAKI, M. A Hybrid Multi-Swarm Particle Swarm Optimization algorithm for the Probabilistic Traveling Salesman Problem. Computers y Operations Research, v. 37, n. 3, p. 432-442, 2010. http://dx.doi. org/10.1016/j.cor.2009.03.004

MARINAKIS, Y., MARINAKI, M.; DOUNIAS, G. Honey bees mating optimization algorithm for the Euclidean traveling salesman problem. Information Sciences, v. 181, n. 20, p. 4684-4698, 2011. http://dx.doi.org/10.1016/j. ins.2010.06.032

MARTI, R. Procedimientos metaheurísticos en optimización combinatoria. Matemátiques, v. 1, n. 1, p. 1-60, 2003.

MLADENOVIĆ, N. et al. A general variable neighborhood search for the one-commodity pickup-and-delivery travelling salesman problem European Journal of Operational Research, v. 220, n. 1, p. 270-285, 2012. http://dx.doi.org/10.1016/j.ejor.2012.01.036

MONTOYA, J.; PATERNINA, C.; FREIN, Y. Minimización del tiempo total de flujo de tareas en una sola máquina: Estado del arte. Ingeniería \& Desarrollo, v. 12, p. 118-129, 2002.

NGUYEN, $\mathrm{H}$. et al. Implementation of an effective hybrid GA for large-scale traveling salesman problems. IEEE Transactions on Systems, Man, and Cybernetics, Part B: Cybernetics, v. 37, n. 1, p. 92-99, 2007. http://dx.doi. org/10.1109/TSMCB.2006.880136

NY, J.; FERON, E.; FRAZZOLl, E. On the dubins traveling salesman problema. IEEE Transactions on Automatic Control, v. 57, n. 1, art. no. 5983407, p. 265-270, 2012.

OHLMANN, J.; THOMAS, B. A compressed-annealing heuristic for the traveling Salesman problem with time windows. INFORMS Journal on Computing, v. 19 , n. 1, p. $80-90,2007$. http://dx.doi.org/10.1287/ ijoc. 1050.0145

PADBERG, M.; RINALDI, G. Optimization of a 532-city symmetric traveling salesman problem by branch and cut. Operations Research Letters, v. 6, p. 1-7, 1987. http://dx.doi.org/10.1016/0167-6377(87)90002-2 
PÉREZ, J. Heurística inspirada en el análisis sistémico del "Vecino más cercano", para solucionar instancias simétricas TSP, empleando una base comparativa multicriterio. 2011. 130 f. Tesis (Magíster en Ingeniería de Sistemas)-Facultad de Minas, Universidad Nacional de Colombia, Medellín, 2011.

QUEVEDO, D.; RíOS, R. Uso de búsqueda Tabú en la solución del problema de asignación cuadrática. Ingenierías, v. 8, n. 48, p. 40-48, 2010.

REINELT, G. TSPLIB. A traveling salesman problem library. ORSA journal on computing, v. 3, n. 4, p. 376-384, 1991.

RíOS, R.; BARD, J. Heurísticas para secuenciamiento de tareas en líneas de flujo. 2010. Disponível em: <http:// yalma.fime.uanl.mx/ roger/work/Papers/article/ article-ciencia-2000.pdf>. Acesso em: 15 fev. 2011.

RODRÍGUEZ, A.; RUIZ, R. The effect of the asymmetry of road transportation networks on the traveling salesman problem. Computers and Operations Research, v. 39, n. 7, p. 1566-1576, 2012. http://dx.doi.org/10.1016/j. cor.2011.09.005

SAVLA, K.; FRAZZOLI, E.; BULLO, F. Traveling salesperson problems for the Dubins vehicle. IEEE Transactions on Automatic Control, v. 53, n. 6, p. 1378-1391, 2008. http://dx.doi.org/10.1109/TAC.2008.925814

SCHAEFFER, E. Computación aleatorizada - probabilidad y algoritmos. RISCE - Revista Internacional de Sistemas Computacionales y Electrónicos, p. 2-4, enero 2009.

SNYDER, L.; DASKIN, M. A random-key genetic algorithm for the generalized traveling salesman problem. European Journal of Operational Research, v. 174, n. 1, p. 38-53, 2006. http://dx.doi.org/10.1016/j. ejor.2004.09.057

STAPLES, M.; NIAZI, M. Experiences using systematic review guidelines. Journal of Systems and Software, v. 80, n. 9, p. 1425-1437, 2007. http://dx.doi.org/10.1016/j. jss.2006.09.046

STUETZLE, T.; HOOS, H. MAX-MIN Ant System and local search for the traveling salesman problem. In: IEEE CONFERENCE ON EVOLUTIONARY COMPUTATION, 1997. Proceedings... ICEC, 1997. p. 309-314.

SU, S.; CAO, X.; ZUO, X. Traveling salesman problems on a cuboid using discrete particle swarm optimization. Lecture Notes in Electrical Engineering, 154 LNEE, p. 404-411, 2012.

VANSTEENWEGEN, P.; SOUFFRIAU, W.; SÖRENSEN, K. The travelling salesperson problem with hotel selection,
Journal of the Operational Research Society, v. 63, n. 2, p. 207-217, 2012. http://dx.doi.org/10.1057/ jors.2011.18

VAZIRANI, V. Approximation algorithms. Corr. 2nd print. New York: Springer-Verlag Inc., 2001. p. 1-380.

VÉLEZ, M.; MONTOYA, J. Metaheurísticos: una alternativa para la solución de problemas combinatorios en administración de operaciones. Revista EIA, n. 8, p. 99-115, 2007.

VERSCHAE, J. Algoritmos de aproximación para problemas de programación de ordenes en maquinas paralelas. Memoria para ingeniero civil matemático. Universidad de Chile, Facultad de ciencias físicas y matemáticas, 2008. p. ix-xiii.

WANG, R.; ZHAO, L.; ZHOU, X. Ant colony optimization with memory and its application to traveling salesman problem. IEICE Transactions on Fundamentals of Electronics, Communications and Computer Sciences, E95-A, no. 3, p. 639-645. 2012.

WEYLAND, D.; MONTEMANNI, R.; GAMBARDELLA, L. Using statistical tests for improving state-of-the-art heuristics for the probabilistic traveling salesman problem with deadlines. Lecture Notes in Computer Science (including subseries Lecture Notes in Artificial Intelligence and Lecture Notes in Bioinformatics), 6927 LNCS (Part 1), p. 448-455, 2012.

WHITLEY, D.; STARKWEATHER, T.; FUQUAY, D. Scheduling Problems and Travelling Salesman: The Genetic Edge Recombination Operator. In: SCHAFFER, J. (Ed.). Proceedings on the Third International Conference on Genetic Algorithms. Los Altos: Morgan Kaufmann Publishers, 1989. p. 133-140.

WU, H.; WU, J.; LIU, A. Hybrid discrete particle swarm optimizer algorithm for traveling salesman problem. Advanced Materials Research, v. 433-440, p. 4526-4529, 2012.

\section{Agradecimientos}

Se agradece a los diferentes evaluadores que contribuyeron al mejoramiento de este artículo, a la Asociación Brasileña de Ingeniería de Producción (ABEPRO), a los profesores de la Universidad Nacional de Colombia, Juan David Velásquez y Luis Fernando Moreno, y al profesor de la Universidad de Antioquia, Oscar Ortega.

\title{
Relevant literary space on travelling salesman problem (TSP): contents, classification, methods and fields of inspiration
}

\begin{abstract}
This paper describes and analyzes, in terms of content, the types of TSP, methods and fields of inspiration - the most relevant "literary space" on TSP. It is built based on the top most cited on the TSP history and the Top 10 cited from 2006 to 2010. The study of TSP still prevails in Research, focusing on the original problem and its variants: Multiple TSP (m-TSP) and Probabilistic TSP (PTSP). Evidence shows that there has been progress in the development of TSP solving methods, highlighted by various inspiration fields: biological evolution, behavior of real ants, thermodynamics, systematic strategies for combining decision rules, and neighborhood search. There is a tendency to develop hybrid methods, in particular by integrating global approaches to local search. There is need to introduce new fields of inspiration.
\end{abstract}

\section{Keywords}

Travelling salesman problem. Systematic review. Heuristic method. Combinatorial optimization. 